\title{
Conhecimento dos professores de Educação Física escolar sobre promoção da saúde
}

\section{Knowledge of school Physical Education teachers on health promotion}

\author{
Bruna Fernanda Justino da Costa $^{1}$, Mateus Dias Antunes ${ }^{2}$, Leonardo Pestillo de Oliveira ${ }^{3}$, \\ Glaukus Regiani Bueno ${ }^{1}$ \\ 1- Faculdade Intermunicipal do Noroeste do Paraná - FACINOR, Loanda, PR, Brasil \\ 2- Universidade de São Paulo - USP, São Paulo, SP, Brasil. \\ 3- Centro Universitário de Maringá - UNICESUMAR, Maringá, PR, Brasil.
}

RESUMO

Objetivo: este estudo teve como objetivo investigar o conhecimento dos professores de educação física escolar em relação à promoção da saúde. Método: o presente estudo se caracteriza por uma análise de pensamentos; nesse sentido foi aplicado a metodologia qualitativa, e para seu mateus_antunes03@hotmail.comdesenvolvimento,o trabalho se adequou por meio de um caráter exploratório descritivo analítico. Para alcançar esse escopo, foi aplicado um questionário sócio demográfico semiestruturado. O experimento didático foi aplicado para todos os professores de educação física do ensino médio das escolas da rede estadual do município de Loanda - PR, totalizando 6 profissionais. Resultados: nas perspectivas das análises sobre a promoção da saúde, notamos resultados condizentes em relação ao seu conceito na essência, porém, desprovidos de uma noção mais aprofundada de alguns conceitos e ações sobre o tema. Alguns aspectos importantes foram evidenciados, como o ensinamento das questões sobre promoção da saúde nas escolas, necessidades de intervenções de capacitação para os profissionais da educação física e planejamento de ações visando a dissipação do conceito de promoção da saúde no ambiente escolar. Conclusão: percebe-se que o

Palavras-chave: Promoção da Saúde; Educação Física; Professores; Empoderamento; Ensino Médio. conhecimento da maioria dos respondentes mostrou-se relevante sobre o tema, entretanto, desprovidos de uma noção mais aprofundada dos conceitos e ações, restringindo-se a experiências focais. Destaca-se a importância de estudos na área para atualizações sobre a promoção da saúde, que contribua efetivamente para o desenvolvimento de capacidades, aquisições, competências e empoderamento dos professores.

\section{ABSTRACT}

Objective: the aim of this study was to investigate the knowledge of physical education school teachers in relation to health promotion. Method: the present study is characterized by an analysis of thoughts. In this sense, the qualitative methodology was applied and, for its development, the work was adapted by means of an analytical descriptive exploratory character. To reach this scope, a semistructured socio-demographic questionnaire was applied. The didactic experiment was applied to all high school physical education teachers of the state schools of the city of Loanda - PR, totaling 6 professionals. Results: in the perspectives of the analyzes on health promotion, we note consistent results regarding its concept in essence, however, lacking a deeper understanding of some concepts and actions on the subject. Some important aspects were evidenced, such as the teaching of health promotion issues in schools, the need for training interventions for physical education professionals and the planning of actions aimed at dissipating the concept of health promotion in the school environment. Conclusion: the knowledge of most respondents

Keywords:

Health Promotion; Physical Education; School teachers; Empowerment; High School. was relevant on the subject, however, devoid of a deeper notion of concepts and actions, restricted to focal experiences. It is worh noting the importance of studies in the area for updates on health promotion, which contributes effectively to the development of capacities, acquisitions, competencies, and empowerment of teachers. 


\section{INTRODUÇÃO}

$\mathrm{Na}$ contemporaneidade ainda surgem algumas dúvidas a respeito do processo de desenvolvimento da saúde e alguns conceitos que as norteiam. Evidenciamos alguns conhecimentos do processo saúde-doença, como a prevenção, reabilitação a promoção da saúde (PS). Promoção da saúde é o processo que visa aumentar a capacidade dos indivíduos e das comunidades para controlarem a sua saúde, no sentido de melhorar e/ou atingir um estado de completo bem-estar físico, mental e social. O indivíduo ou grupo devem estar aptos a identificar e realizar as suas aspirações, a satisfazer as suas necessidades e a modificá-las ou adaptá-las ao meio. Assim, a saúde é entendida como um recurso para a vida e não como uma finalidade de vida ${ }^{1}$.

Algumas condições são fundamentais e necessárias para a atingir a saúde, por isso o Ministério da Saúde(MS), lançou a Política Nacional de Promoção da Saúde (PNPS) com o objetivo de promover a qualidade de vida e reduzir a vulnerabilidade e risco à saúde, aspectos relacionados aos seus determinantes e condicionantes modos de viver, condições de trabalho, habitação, ambiente, educação, lazer, cultura, acesso a bens e serviços essenciais ${ }^{2-4}$.

Em uma perspectiva interdisciplinar, a promoção da saúde vislumbra a melhoria das condições de vida da população e o reconhecimento do direito de cidadania, tendo como princípios a concepção holística da saúde, a equidade, a intersetorialidade, a participação social, sustentabilidade e o meio educacional $^{2}$. Nesse eixo educacional, a escola é considerada como um ambiente protetor da saúde e, segundo a Organização Panamericana da Saúde (OPAS), a promoção da saúde na escola é prioridade. É considerada uma instituição onde o jovem passa grande parte da vida e pode contribuir na construção de valores no qual a saúde está incluída primariamente. É importante o desenvolvimento de um processo dialógico, problematizado e inclusivo, que oportunize ao aluno a construção da consciência crítica sobre si próprio e seu estar no mundo 5 .

A educação em saúde nas escolas auxilia os indivíduos a pensarem formas de viver que os protejam, discutindo comportamentos e criando espaços de reflexão sobre hábitos saudáveis de vida. A escola deve estar voltada para a busca de uma educação integral, desenvolvendo habilidades pessoais que contribuam para a criação e manutenção de ambientes saudáveis e protetores. As ações devem reduzir a exposição a fatores de risco de doenças e agravos não transmissíveis, bem como reforçar fatores de proteção relacionados ao estilo de vida, integrando e estimulando o fortalecimento das relações homem/ ambiente, objetivando contribuir para uma melhor saúde e bem estar, reforçando a capacidade dos indivíduos e comunidades para o enfrentamento das situações de vida ${ }^{5}$.

Nas diretrizes curriculares da educação básica, no título diretrizes curriculares da disciplina de educação física, é abordado que devem ser trabalhados alguns elementos, entre eles, a cultura corporal e saúde, sendo assim, a disciplina de educação física tem tanto responsabilidade quanto contribuição para que seja desenvolvido esse trabalho com a promoção da saúde ${ }^{6}$.

Assim, se faz necessário a inclusão do profissional de educação física capacitado para colaborar com o trabalho de promoção da saúde na escola. Diante do elemento da cultura corporal e da saúde, fica claro a necessidade e implementação de trabalhos de promoção da saúde na escola com profissional de educação física. Diante desse contexto, nossa pesquisa se faz importante por compreender e descrever o atual conhecimento dos professores de educação física sobre o tema promoção da saúde. Com esse diagnóstico e da nova perspectiva da realidade educacional, novos trabalhos podem ser realizados visando melhorias, com atuações diretas sobre os problemas existentes relacionados à promoção da saúde nas escolas.

Essa pesquisa nos dá subsídios para que possamos identificar o atual conhecimento dos professores sobre o tema. Uma ausência de estudos dessa categoria, não nos contemplaria em novos conhecimentos, nesse sentido, o aluno não desfrutaria da coletividade da escola com seus professores envolvidos para futuras ações de promoção da saúde. Com professores não atualizados ou sem conhecimento adequado, não seria possível a capacitação dos alunos e da sociedade em geral, o que levaria o menor controle e identificação da sua saúde, no sentido de melhorar o seu estado de bem estar geral. Professores bem preparados, promovem autonomia, criatividade, participação e capacitação holística do estado geral da saúde.

Este estudo busca fornecer subsídios para futuras ações no âmbito educacional, cultural, social e da saúde para estudantes do ensino médio com 
extensão à população em geral. Portanto, o objetivo do presente estudo foi investigar o conhecimento dos professores de educação física escolar em relação à promoção da saúde.

\section{MÉTODO}

O presente estudo se caracteriza por uma análise de pensamentos, nesse sentido foi aplicado a metodologia qualitativa, e para seu desenvolvimento, o trabalho se adequou por meio de um caráter exploratório descritivo analítico, mostrando-se mais adequado, pois não transforma os pensamentos em números através de porcentagens, o que poderia levar à perda de muitas informações relevantes.

A coleta de dados foi realizada no período de abril a maio de 2017, dentro do universo das quatro escolas estaduais de ensino fundamental da cidade de Loanda, situada no noroeste do Paraná. No primeiro momento foi aplicado um questionário sociodemográfico semiestruturado para o conhecimento do perfil e do tema acerca da promoção da saúde dos professores de educação física das escolas estaduais do ensino médio. Após essa etapa, os dados foram transcritos usando a ferramenta Microsoft Word, analisados e interpretados conforme o código de ética de pesquisa, comparadas e referenciadas com a literatura atual por meio de artigos científicos, teses, dissertação, cartas (documentos de conferências nacionais e internacionais) sobre promoção da saúde.

Como critérios de inclusão deste estudo foram selecionados apenas os profissionais de educação física, de ambos os sexos, do ensino médio dos colégios estaduais. Foram excluídos da amostra os professores que não se enquadraram na mesma formação acadêmica devido à abordagem do tema proposto pelo estudo a ser realizado e também professores do ensino fundamental. Os professores assinaram o termo de consentimento livre e esclarecido (TCLE) para participarem do estudo.

Antes da execução da pesquisa, foi solicitado o documento de autorização local na secretaria de cada escola participante. Considerando o exposto e os objetivos propostos neste estudo, optou-se por elaborar um roteiro semiestruturado de entrevista com questões abertas, suficientemente para captar os conhecimentos acerca do tema promoção da saúde de uma forma descritiva. Esses instrumentos permitem que os entrevistados se expressem livremente, produzindo um discurso ${ }^{7}$.

As perguntas foram as seguintes:

- Qual o seu conhecimento sobre a promoção da saúde?

- Como a promoção da saúde pode ser ensinada nas aulas de educação física?

- A equipe diretiva pedagógica de sua escola promove reuniões para o planejamento de ações promotora da saúde com os professores de educação física? Justifique?

- A escola promove palestras com multiprofissionais sobre a promoção da saúde? Se sim, quais?

- Você já participou de uma formação de conhecimento sobre ações para a promoção da saúde na escola?

Após as coletas de dados foram realizados as análises de dados em relação às repostas de cada um dos professores.

\section{RESULTADOS E DISCUSSÃO}

\section{Reflexões acerca da promoção da saúde pelos professores de educação física das escolas do ensino fundamental}

Os dados foram coletados entre os professores de educação física da rede estadual do município de Loanda-PR. Os professores questionados (P1: professor 1 a P6: professor 6) são nascidos entre os anos de 1962 a 1977, com idades entre 40 a 55 anos. Dos 6 profissionais questionados, quatro são do sexo masculino e dois do sexo feminino. A conclusão de formação acadêmica dos professores varia entre o período de 1988 a 2003, já o tempo de atividade em sala de aula nas escolas, variam de 14 a 29 anos. Dentre os selecionados, a formação acadêmica mais elevada foi a titulação de mestrado.

\section{Conhecimento dos professores de educação física} sobre promoção da saúde.

A promoção da saúde é um processo que consiste em proporcionar para as pessoas os meios 
necessários para melhorar sua saúde, exercendo maior controle sobre ela. E até mesmo como a soma das ações da população, dos serviços de saúde, das autoridades sanitárias, setores sociais e produtivos, voltados para o desenvolvimento de melhores condições de saúde individual e coletiva ${ }^{3}$. Essa descrição reflete o relato do respondente:

\section{P1[...] Defino como a capacidade das pessoas e da comunidade em geral poder modificar os determinantes da saúde em beneficio da própria qualidade de vida $e$ é claro que com políticos programas voltados para isso.}

Em consonância com a Carta de Ottawa, a promoção da saúde não é uma responsabilidade exclusiva do setor da saúde, pois exigem estilos de vida saudáveis para atingir o bem estar, e os prérequisitos para a saúde são: paz, abrigo, educação, alimentação, recursos econômicos. Sendo assim, fica claro que a educação promove saúde ${ }^{1}$. É o que vemos nas descrições de P2, pois sua fala corrobora com a literatura e vislumbra o processo educacional como primordial a melhores estados de promoção da saúde:

\section{P2 [...] A promoção da saúde é muito importante, principalmente na área escolar. Tenho noções básicas onde posso passar e até mesmo atender basicamente em algum caso. Tento passar para os alunos as diversas formas de promover a saúde.}

A promoção da saúde pretende reduzir as desigualdades existentes nos níveis de saúde das populações e assegurar a igualdade de oportunidades e recursos, com vista a capacitá-los para completa realização do seu potencial de saúde. Para atingir este objetivo, torna-se necessária uma sólida implantação num meio favorável, acesso à informação, estilos de vida e oportunidades que permitam opções saudáveis. As populações não podem realizar totalmente o seu potencial de saúde sem que sejam capazes de controlar os fatores que a determinam. Este princípio deve aplicar-se igualmente às mulheres e aos homens ${ }^{1}$.

Nas respostas de P3 e P4 evidenciamos que a compreensão da pergunta realizada foi de acordo o conhecimento subjetivo do entrevistado, e não exemplificando com conceitos, definições e exemplos práticos de si mesmo em relação ao caráter de promoção da saúde, subjetivando as respostas e suas evidências de conhecimento.

\section{P3 [...] Entre possiveis formas de} classificar meu conhecimento sobre promoção da saúde considero meu conhecimento bom em uma escala organizada de como: RUIM, REGULAR, BOM, EXCELENTE, mas essa é a minha auto avaliação e pode não corresponder à realidade.

P4 [...] Conhecimento básico, não de formação (graduação) necessariamente aproveitando conteúdos dofuncionamento do corpo humano da adequação adaptando a informações atualizadas através da internet.

Nos últimos dois entrevistados, conseguimos evidenciar que as respostas vão ao encontro de um adequado conceito de promoção da saúde, no entanto, a promoção da saúde não se caracteriza apenas em atividades físicas, bens matérias, lazer e cultura. Heidmann et $a l .{ }^{4}$ destacam que as dimensões de promoção da saúde vão além de questões física e descrevem cinco estratégias para atingir a promoção de saúde de uma população, em especial no âmbito educacional. São elas: as políticas públicas, favorecimento de ambientes saudáveis, o apoio da ação comunitária, reorientação do serviço de saúde e concepção de habilidades pessoais.

\section{P5 [...] Quanto à importância da alimentação equilibrada, com balanço energético. Valor calórico dos exercícios e gastos calóricos nas atividades físicas $e$ as devidas atividades para a promoção da saúde e qualidade de vida.}

P6 [...] Promover a saúde, compreendo e tenho noções de qualidade de vida, acesso aos bens materiais, culturas e lazer.

\section{Como a promoção da saúde pode ser ensinada nas aulas de educação física.}

Orfei e Tavares $^{8}$ destacam que o professor 
de educação física deve desenvolver em suas aulas, métodos para contribuir com a promoção da saúde do aluno como: avaliações antropométricas, avaliação do nível de aptidão física, atividades físicas de forma geral e promover experiências motoras. São ações que proporcionam repercussão satisfatória em direção a um melhor estado de saúde, afastando ao máximo a possibilidade de aparecimento dos fatores de risco que contribuem para o surgimento de eventuais distúrbios orgânicos e sociais.

Diante desse contexto, o papel da educação física nas escolas deve incluir a capacitação dos alunos a controlar a sua saúde e propiciar atividades físicas que os preparem para ter o corpo sadio. Promover reuniões com os profissionais da instituição, para o planejamento de ações para capacitar os indivíduos a autopromover saúde e dissipar o conhecimento em suas atividades, também são necessárias. Nesse cenário, as aulas de educação física devem ser preparadas para que esses objetivos sejam atendidos.

As considerações de P1,P6 foram semelhantes e atribuem circunstâncias importantes de conhecimento e atitudes a serem inseridas como promoção da saúde nas aulas de educação física.

\section{P1[...] Através de textos, debates, atividades corporais e escritas, seminários, vídeos. \\ P6[...] Através de aulas teóricas e práticas.}

As declarações dos professores correspondem ao conceito de Rodrigues ${ }^{9}$ que relata que as atividades desenvolvidas são: recreativas, pré-esportivas, jogos, brincadeiras, massagens, alongamento, cantigas de rodas, atividades aeróbicas que se referem principalmente a caminhada e ginástica de baixo impacto, atividade de força que consiste em exercícios em circuito, alteres e bastões. Também faz parte das atividades a avaliação física, medidas de circunferências, dobras cutâneas, peso, altura e controle da pressão arterial.

As descrições de P2, P4, P5, sobre como a promoção da saúde pode ser ensinada nas aulas de educação física, não faz junções às perspectivas atuais, apresentando-se de forma equivocada e não holística, e fica claro que a consciência de promoção da saúde pelos relatos, evidencia a preocupação com as atividades físicas específicas e não o indivíduo como um todo e a soma de suas partes.

P2[...] Através das aulas teóricas, com explicações básicas, de como se promove a saúde, incluindo o bem entre o aluno.

P4[...] Através de aulas teóricas demonstrando os beneficios da atividade física para manutenção da saúde, esclarecendo o que acontece no organismo e também mostrando os malefícios da falta de atividade.

\section{P5[...] Através de atividades teóricas} desenvolvidas em salas, vídeos $e$ atividades práticas desenvolvidas durante as aulas.

No estudo de Orfei e Tavares ${ }^{8}$ fica evidente que os conteúdos a serem ensinados são: palestras e debates sobre a promoção da saúde, atividade física, alimentação saudável, uso de álcool e o tabagismo, sexualidade, entre outros, a fim de conscientizá-los sobre boas práticas. É importante consciencializar os alunos a tomarem iniciativa em relação à prática de atividades físicas e hábitos saudáveis para que se tornem ativos fisicamente, não apenas na infância e na adolescência, mas também na idade adulta. Além disso, projetos sobre atividade física, alimentação saudável, postura, entre outros, nos quais todos os estudantes, funcionários e comunidade escolar participem, também devem ser realizados.

De acordo com Cardoso, Reis e Iervolino ${ }^{10}$ é necessário uma combinação de ação social planejada e de experiências de aprendizagem, visando capacitar os indivíduos para adquirirem controle sobre o comportamento em saúde, e sobre as condições sociais que afetam seu próprio estado e o estado de saúde dos outros.

Orelato de P3 deixa claro que não compreendeu a questão, desviando do assunto.

P3 [...] Considerando que as aulas de educação fisica podem contribuir para que o aluno possa melhorar seu conhecimento sobre avaliação entre aptidão física e saúde, agora se vão mudar seu comportamento é uma outra questão. 


\section{A equipe diretiva pedagógica de sua escola promove reuniões para o planejamento de ações promotora da saúde com os professores de educação física. Justifique.}

Novas concepções estão surgindo a partir de contribuições de profissionais e pesquisadores da área, estimulando a elaboração de novas estratégias, em geral pautadas nas mais recentes concepções de saúde e qualidade de vida. Dentre as ações específicas priorizadas pela PNPS, criada em 2006, inclui-se a prática corporal e atividade física (PCAF) nas ações de rede básica de saúde e na comunidade, fundamentando a inserção do profissional da educação física no serviço de atenção básica ao compor equipes para o planejamento de ações ${ }^{11}$.

A ação intersetorial precisa ser negociada e incluída na rotina e na prática dos profissionais, permitindo construção de saberes e mais diálogos na contextualização de políticas de saúde nas escolas. Parcerias e ações intersetoriais são efetivas quando reúnem e dialogam com a pluralidade de atores institucionais e não institucionais envolvidos e interessados. Nesse sentido, os programas de saúde na escola ainda têm muito que caminhar para avançar em uma perspectiva mais integradora e inovadora ${ }^{12}$.

Faz-se necessário uma demanda de mediação, desenvolvimento de ação intersetorial, por parte dos professores e das instituições envolvidas, em torno de planos territoriais. A intersetorialidade proporciona a compreensão multidimensional e ampla da problemática acerca da atenção em saúde, em todos os níveis, implicando em intervenções de diversos setores, articulando diferentes práticas e saberes com $\mathrm{o}$ intuito de desenvolver ações produtivas e eficazes ${ }^{13}$.

Os relatos dos professores quando questionados se participam de reuniões para ações de promoção da saúde nas escolas em que atuam são:

\section{P1[...] Raramente.}

\section{P2[...] Não.}

P3[...] Não, falta de organização, falta de tempo, falta de hábito, falta de conhecimento.

$$
\text { P4[...] Não. }
$$

P5[...] Não, o professor que desenvolve seu planejamento anual e desenvolve ações promotora a saúde do educando.

\section{P6[...] Através de palestras com técnicas de saúde.}

Diante dos dados coletados percebe-se que o profissional de educação física não está participando de reuniões para o planejamento de ações promotoras da saúde nas escolas, demostrando uma realidade negativa do ponto de vista da promoção da saúde. Diante dessa atual realidade, é necessário a interação de todos os envolvidos, ou seja, a organização com os profissionais das instituições que podem contribuir para o processo que visa aumentar a capacidade dos indivíduos e da comunidade a terem o autocontrole da saúde, por meio de ações específicas a essa população.

\section{A escola promove palestras com multiprofissionais sobre a promoção da saúde. Se sim, quais.}

A escola promotora da saúde deve ser inclusiva e garantir a participação efetiva de todos os envolvidos, com objetivo de criar atitudes, ambientes mais saudáveis, desenvolvendo habilidade e estimulando a tomada de decisão por meio da coresponsabilização em promoção da saúde ${ }^{10}$.

A escola higiênica, por exemplo, funciona com hábitos higiênicos e contribui para educação da população. As crianças adquirem bons costumes e os propagam em casa, onde desejam que tudo se passe como na escola, cujo meio puro e sadio facilmente se acostuma. Promoção de saúde na escola prioriza aspectos específicos a serem abordados como: alimentação saudável, prática de atividade física, alertas sobre o uso de tabacos, drogas sintéticas e sexualidade, dentre outros determinantes de saúde ${ }^{12}$.

Os relatos de P1, P2, P3, P5 e P6 nos evidenciam que as palestras com multiprofissionais são feitas nas escolas, com aparecimento maior do profissional da saúde (enfermeiros) que enfatizam temas como: doenças e ações curativas. Porém, no âmbito da promoção da saúde deveríamos evidenciar ações profiláticas em maior escala.

Adiante, a descrição dos relatos dos professores:

P1 [...] Sim, DST (doenças sexualmente transmissíveis), doenças crônicas, alimentação saudável. 
P2 [...] sim o básico, através de profissionais diversos da área. (médico, dentistas, enfermeiros).

P3 [...] sim palestras com profissionais da área da saúde da UNIPAR/EAD (universidade Paranaense e a distância) e FACINOR (faculdade intermunicipal do noroeste do Paraná) dos cursos de educação física e enfermagem.

P5[...] simpromoveaçõescompalestrantes de enfermagem, com palestras de médicos e outros profissionais que promovem a área da saúde do educando.

P6 [...] abordam palestras sobre a prevenção de uso de ações, DST (doenças sexualmente transmissiveis) e drogas licitas como: fumo e álcool.

Já o professor P4 relata que:

\section{[...] a escola não promove palestras com multiprofissionais sobre a promoção da saúde.}

Conforme descrito anteriormente pelos cinco professores, as atividades com temas ligados à saúde já eram realizados no município, e isso vem acontecendo por meio de palestras realizadas nas escolas por profissionais da área da saúde.

Fica claro que a maioria das escolas promove palestras com multiprofissionais sobre a promoção da saúde, mas devido aos conteúdos abordados e certa escassez de profissionais, isso se torna insuficiente e poderia ser mais abrangente, tais como a inclusão de outros profissionais e mais temas relacionados à saúde. Profissionais tais como médicos, professores de educação física, biologia, dentre outros, podem atuar por meio de palestras para os alunos, abordando temas como as prevenções e cuidados com a saúde, exercícios físicos e alimentação saudável.

Além da integração educacional Fracoli et al. ${ }^{14}$ relatam que a promoção da saúde deve compreender mais ações, dentre elas: ação intersetorial, parcerias com creches, instituiçõs de longa permanência, escolas, comerciantes, grupos sociais e ações de inclusão social. Dentre esses aspectos, também existe a orientação da família e ou portador de necessidades especiais quanto às medidas facilitadoras para sua máxima inclusão social.

\section{Você já participou de uma formação de conhecimento sobre ações para a promoção da saúde na escola. Se sim, quais.}

É de grande relevância salientar que a formação continuada não pode ser vista apenas como a participação em cursos e seminários. Estes eventos são importantes, mas estão em um modelo horizontal de transmissão de conhecimento, não ocorrendo diálogo. Formação continuada não é simplesmente acumular informações. A preocupação de formação continuada deve proporcionar a volta aos estudos, pesquisas sobre teorias e novas práticas pedagógicas, ou seja, inovações. Essas inquietudes no processo de conhecimento são alternativas para administrar a efervescência das diversidades e desigualdades sociais $^{15}$.

O professor nesse contexto, deve estar em busca constante pela formação continuada, sendo seu dever, manter-se atento de que esse processo de conhecimento não é finito, e estar consciente das necessidades de atualizações, proporcionado maiores capacidades críticas e de reconstrução da prática educativa. A inovação é toda tentativa consciente de introduzir uma mudança no sistema de ensino com a finalidade de melhorá-lo. Essa mudança poderá ser alcançada a partir do momento em que o professor perceber-se como ator principal e das necessidades de formação adequada para a docência.

Temp et al. ${ }^{15}$ ainda contemplam que umas das funções do professor é buscar seu aprimoramento teórico e prático para cumprir seu papel de mediador entre o conhecimento sistematizado, os saberes da prática social e a cultura na qual acontece o ato educativo, incluindo as estruturas sociocognitivas do aluno. Assim, mediante essa ação reflexa, o professor coloca-se em um processo constante de recriação de si próprio. Ainda é relevante destacar que o professor necessita reconstruir sua prática através da ligação entre novas e antigas teorias com sua ação de trabalho, formado um novo modelo de ensino. Os educadores, incluindo docentes universitários, são pessoas que também estão em desenvolvimento e que precisam continuamente educar-se para poder educar ${ }^{16-21}$.

Na perspectiva de Cardoso, Reis e Iervolino ${ }^{10}$ o professor exerce uma influência constante e ativa 
sobre os conceitos de saúde e doença dos seus alunos. Sua sensibilidade e didática devem dar condições de repassar informações acerca da saúde e adaptá-las ao ambiente escolar, necessitando de um suporte na área para subsidiar o seu trabalho. Geralmente o professor torna-se referência para os alunos e pode estimular a compreensão e adoção de hábitos saudáveis, além disso, um professor preparado para observar corretamente o ambiente escolar e perceber os riscos, pode proteger a saúde dos escolares e seus familiares.

$\mathrm{Na}$ sequência, evidenciamos que os relatos dos professores, exceto um respondente, mostram que já participaram de palestras e reuniões para formação de conhecimento, porém, nenhuma espécie de curso mais elevado na área.

O núcleo Regional de Ensino do município de Loanda - PR promove palestras para a capacitação dos profissionais com atividades a promoção da saúde do Educando, conforme relatado por:

\section{P5. P1 [...] sim, palestras.}

P2 [...] não só através de palestras na escola com profissionais.

P3 [...] sim nessa escola participo de reuniões sobre DST (doenças sexualmente transmissiveis).

P4 [...] não.

P5 [...] sim, no curso de educação física do núcleo regional de ensino (NRE) atividades a promoção da saúde ao educando.

P6 [...] não.

\section{CONCLUSÃO}

Ao analisarmos o conteúdo, nos faz refletir cada vez mais, que o ser humano nunca está completo cognitivamente, estando sempre em construção de novos conhecimentos. Assim, entendemos que mudanças importantes ocorrem a partir da sua interação com o meio, buscando uma formação continuada, ampliando e atualizando seus conhecimentos.

Nas perspectivas das análises sobre a promoção da saúde, notamos resultados condizentes em relação ao seu conceito na essência, porém, desprovidos de uma noção mais aprofundada de alguns conceitos e ações sobre o tema. Alguns aspectos importantes foram evidenciados, como o ensinamento das questões sobre promoção da saúde nas escolas, necessidades de intervenções de capacitação para os profissionais da educação física e planejamento de ações, visando a dissipação do conceito de promoção da saúde no ambiente escolar.

Contudo, entendemos que é necessário uma formação continuada e específica para um aprofundamento da aplicação dos conteúdos de promoção da saúde e maior participação do profissional de educação física, fora do âmbito das salas de aula, como reuniões de planejamento de ações promotoras de saúde. A promoção da saúde, quando ensinada na escola, deve ser uma ação constante, devendo contribuir no desenvolvimento de capacidades, aquisições e competências de cada indivíduo e da comunidade, tendo como foco desenvolver hábitos saudáveis e possibilitar o pleno exercício da cidadania. O ambiente escolar deve ser cenário de transformações e mudanças de comportamento, melhorando a interação do indivíduo com o coletivo.

\section{REFERÊNCIAS}

1. Carta de Ottawa. Primeira conferência internacional sobre promoção da saúde. Ottawa, novembro de 1986.

2. Silva RM, Araujo MAL. Promoção da saúde no contexto interdisciplinar. Revista Brasileira em Promoção da Saúde. 2007;20(3):141-142.

3. Derntl AM, Watanabe HA. Promoção da saúde. Litvoc J, Brito FC. Envelhecimento: prevenção e promoção da saúde. São Paulo: Atheneu, p. 37-45, 2004.

4. Heidmann ITSB, Almeida MD, Boehs AE, Wosny ADM, Monticelli M. Promoção à saúde: trajetória histórica de suas concepções. Texto \& Contexto - Enfermagem. 2006;15(2):352358. doi: http://dx.doi.org/10.1590/S0104-07072006000200021 5. Bernardes AG, Hillesheim B, Souza EO, Marques CF. Psicologia e Regimes de verdade nas práticas de promoção da saúde. Fractal. 2016;28(1)2-8. doi: http://dx.doi. org/10.1590/1984-0292/1027

6. Paraná SEED. Diretrizes Curriculares da Educação Básica-. Língua Portuguesa. Paraná, 2008.

7. Lefevre F, Lefevre AMC. Promoção de saúde: a negação da negação. Rio de Janeiro: Vieira e Lent, 2004.

8. Orfei JM, Tavares VP. Promoção da saúde na escola através das aulas de Educação Física. Alimentação, Atividade Física e Qualidade de Vida dos Escolares do Município de Vinhedo/SP, p. 81, 2009.

9. Rodrigues ZM, Costa DS, Barros JB. Educação Física e Promoção da Saúde: Contribuição para o SUS. In IV Congresso Centro-Oeste de Ciências do Esporte e I Congresso Distrital de 
Ciências do Esporte, 2010.

10. Cardoso V, Reis AP, Lervolino SA. Escolas promotoras de saúde. Revista Brasileira de Crescimento e Desenvolvimento Humano. 2008;18(2):107-115. doi: http://dx.doi.org/10.7322/ jhgd.19872

11. Scabar TG, Pelicioni AF, Pelicioni MCF. Atuação do profissional de Educação Física no Sistema Único de Saúde: uma análise a partir da Política Nacional de Promoção da Saúde e das Diretrizes do Núcleo de Apoio à Saúde da Família-NASF. J Health Sci Inst. 2012;30(4):411-418.

12. Silva CDS, Bodstein RCD. A. Referencial teórico sobre práticas intersetoriais em Promoção da Saúde na Escola. Ciência \& Saúde Coletiva. 2016;21(6):1777-1788. doi: http://dx.doi. org/10.1590/1413-81232015216.08522016

13. Tavares MDFL, Rocha RMD, Bittar CML, Petersen CB, Andrade MD. A promoção da saúde no ensino profissional: desafios na Saúde e a necessidade de alcançar outros setores. Ciência \& Saúde Coletiva. 2016;21(6):1799-1808. doi: http:// dx.doi.org/10.1590/1413-81232015216.07622016

14. Fracoli AL, Gomes PMF, Rodrigues ZNF, Oliveira AJ, Pacheco SRC, Tatiane FB. Perspectivas das ações intersetoriais, de inclusão e participação sociais desenvolvidas por agentes comunitários de saúde. Revista Brasileira de Promoção de Saúde. 2015;28(1):82-88. doi: http://dx.doi. org/10.5020/18061230.2015.p82

15. Temp H. Formação Continuada no Ensino Superior: um estudo com professores que atuam em Cursos de Educação Física, 2013.

16. Mosquera JJM, Stoubäus CD. auto-imagem, auto-estima e auto-realização na universidade. In: ENRICONE, D. (Org). A docência na educação superior- sete olhares. 2 ed. Porto Alegre: EDIPUCRS, 2008.

17. Bydlowski CR, Lefèvre AMC, Pereira IMTB. Promoção da saúde e a formação cidadã: apercepção do professor sobre cidadania. Ciência \& Saúde Coletiva. 2011;16(3):1771-1780. doi: http://dx.doi.org/10.1590/S1413-81232011000300013

18. Gomes JP. As Escolas Promotoras de Saúde: uma via para promover a saúde e a educação para a saúde da comunidade escolar. Educação. 2009;32(1):84-91.

19. Guimarães G, Aerts D, Câmara SG. A escola promotora da saúde e o desenvolvimento de habilidades sociais. Diaphora. 2014;12(2):88-95.

20. Moura JBVS. Perspectiva da epidemiologia histórica e a escola promotora da saúde. Revista História, Ciência e Saúde. 2007;4(2):489-501. doi: http://dx.doi.org/10.1590/S010459702007000200006

21. Ribeiro RM, Tribess S, Santos ASD, Pinto LLT, Ribeiro MDCL, Roza LB, et. Barriers to the involvement of the elderly in public services to promote physical activity. Ciencia \& Saúde Coletiva. 2015;20(1):739-749. doi: http://dx.doi. org/10.1590/1413-81232015203.03702014

Como citar: COSTA, Bruna Fernanda Justino da et al. Conhecimento dos professores de Educação Física escolar sobre promoção da saúde. Revista Interdisciplinar de Promoção da Saúde, Santa Cruz do Sul, v. 1, n. 3, set. 2018. ISSN 2595-3664. Disponível em: <https://online.unisc.br/seer/index.php/ripsunisc/article/view/12940>. Acesso em: 07 jan. 2019. doi:https://doi.org/10.17058/ rips.v1i3.12940.

Rev. Interdisciplin. Promoç. Saúde - RIPS, Santa Cruz do Sul, 1(3): 169-177, jul/set. 2018 ISSN: 2595-3664 